\title{
La revitalisation urbaine : l'action publique locale peut-elle vraiment dépasser le « mur » de l’intégration ?
}

\author{
Gérard Divay ${ }^{1}$ \\ INRS-UCS
}

\section{Les caractéristiques de la revitalisation urbaine intégrée}

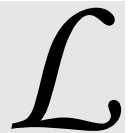
es quartiers défavorisés des villes ont, dans plusieurs pays, fait l'objet, au cours des années 1990, d'une attention politique particulière, parfois avivée par des explosions de violence. Ces quartiers peuvent être situés au centre comme en périphérie des agglomérations. Même s'ils sont plus ou moins hétérogènes, ils concentrent surtout des pauvres et cumulent, à des degrés divers, des handicaps, mesurés comme des écarts à la moyenne d'agglomération, sur plusieurs plans (faible qualité des logements et du milieu physique, accessibilité réduite aux opportunités urbaines, chômage, état de santé, etc.). Ils ont reçu des appellations différentes dont plusieurs (par exemple «en crise», « sensibles», «prioritaires »...) témoignent des inquiétudes et des préoccupations qu'ils suscitent parmi les élites urbaines, sinon dans toute la population.

Leur situation a occasionné de multiples initiatives prises par des groupes communautaires ou par les pouvoirs publics. Des politiques nationales ont été mises de l'avant; par exemple, en France la Politique de la Ville, en Angleterre la Neighborhood Renewal Strategy, en Allemagne la Sozialestadt. Les expériences européennes qui s'inscrivent dans le courant du développement social urbain ont fait l'objet de nombreuses études comparatives et de quelques bilans. Au Québec, des quartiers semblables ont connu un foisonnement d'initiatives communautaires qui s'apparentent aux expériences de développement social urbain, comme le soulignent Jacques L. Boucher et Louis Favreau ${ }^{2}$. Des villes ont aussi lancé des opérations qu'elles qualifient de revitalisation urbaine intégrée et qui mettent à contribution des programmes gouvernementaux sans qu'il n'y ait de politique spécifique des gouvernements supérieurs sur ce thème ${ }^{3}$.

Par delà les différences nationales, ces diverses interventions se ressemblent sur un certain nombre de caractéristiques dont la présence est plus ou moins accentuée et variable dans le temps. Pour Quartiers en crise, il s'agit d'approches "globales », " transversales et interpartenariales »; ces approches sont aussi territorialisées ${ }^{4}$. En synthèse du projet de recherche UGIS, les auteurs notent des «striking similarities between (emerging) concepts and processes (globalisation, social exclusion, social cohesion), problem description (high unemployment, ethnic tensions, ageing populations, unemployment), policy rhetoric and objectives (increasing sustainibility and social cohesion) and policy techniques (area-based policies, co-opération, networking, participation) $»^{5}$. Dans une autre étude comparative européenne, Geddes et Benington font ressortir les thèmes convergents de « social exclusion », « partnership » et « local governance $»^{6}$.

La notion d'intégration se présente comme un leitmotiv dans toutes ces expériences; elle caractérise autant les résultats attendus que la démarche d'action publique préconisée pour les atteindre. Les résultats attendus se situent sur deux plans qui sont intimement liés dans les démarches, sans que leurs interactions ne soient toujours explicitées ${ }^{7}$ : le quartier et les personnes qui vivent dans ces quartiers, notamment les pauvres.

L'intégration des personnes est plus souvent explicitée sous les termes d'inclusion sociale, de réinsertion 
ou encore «d'activation » et vise toutes les sphères de la vie. Il s'agit surtout de la réintégration sur le marché du travail. De nombreuses mesures visent l'employabilité, la création d'emplois ou l'accès aux emplois pour les chômeurs ou les travailleurs à statut précaire de ces quartiers. Mais des efforts sont aussi mis de l'avant pour développer l'intégration sociale, au sens large, de l'amélioration des comportements de civilité à l'insertion dans des réseaux et au renforcement du capital social individuel. On s'attend, en outre, sur un plan plus politique, à ce que les citoyens de ces quartiers participent intensément au processus de revitalisation, à la définition des projets ainsi qu'à leur mise en œuvre.

\section{Si les résultats de la revitalisation se placent sous le signe de l'intégration, la démarche l'est encore plus.}

L'intégration de ces quartiers à la ville a plusieurs dimensions. Souvent ces quartiers sont enclavés physiquement et habituellement stigmatisés socialement. L'ambition de la revitalisation urbaine est de mieux les raccorder au reste de la ville en aménageant les barrières physiques, en améliorant l'accessibilité en général et au besoin en modifiant substantiellement le cadre bâti (modification de la grille de rue et/ou démolition-reconstruction). Mais elle cherche plus globalement à modifier l'image de ces quartiers, notamment en diversifiant la composition sociale et en décloisonnant le marché résidentiel local. Comme ces quartiers de pauvreté concentrée sont supposés handicaper leurs résidents dans leurs chances de réussite économique et sociale, la revitalisation intégrée cherche à briser le moule dans ses dimensions économique et sociale.

L'intégration du quartier touche aussi au plan politique, avec plus d'ambiguïté. Certes, l'empowerment du quartier est valorisé, tout comme la participation des citoyens. Mais la recherche d'une prise en charge des problèmes et des transformations du quartier par les résidents doit composer avec les institutions et les politiques en place. Elle apparaît parfois moins comme un résultat attendu pour le long terme que comme un moyen utile pendant la phase de transformation, qu'on pourrait aussi qualifier de "normalisation", puisque la revitalisation vise à ce qu'en bout de ligne, le quartier soit moins différent sur tous les plans.
Si les résultats de la revitalisation se placent sous le signe de l'intégration, la démarche l'est encore plus. Elle tente de mobiliser l'ensemble du système local d'action publique sous ce qu'on peut appeler une maxime des $5 \mathrm{M}$. La revitalisation urbaine intégrée se veut à la fois multisectorielle, multisphères, multiéchelons, multiniveaux et multipartenaires.

- Multisectorielle : des interventions sont mises de l'avant dans plusieurs secteurs ou domaines : le cadre bâti, la formation, l'éducation, la sécurité, l'emploi, la culture... et si possible, chaque intervention sectorielle tient compte des autres, voire les suscite.

- Multiéchelons : la revitalisation urbaine intégrée concentre ses projets sur de petits territoires, d'ailleurs de tailles très disparates. Mais en même temps, elle suppose ou appelle des interventions à une échelle plus vaste, la ville ou l'agglomération, pour des raisons évidentes; les problèmes d'emploi ne se règlent pas au niveau d'une unité de voisinage et l'amélioration de l'accessibilité passe souvent par une action sur tout le réseau.

- Multisphères : la revitalisation urbaine ne mise pas seulement sur les mesures gouvernementales et les interventions des organismes publics; elle met largement à contribution les initiatives du monde associatif et communautaire et tente d'intéresser les entreprises privées. Les sphères publiques, privées et associatives sont donc conviées au projet collectif local.

- Multiniveaux d'organisation sociale: les actions touchent les individus (il faut les intégrer en développant leurs atouts), les organisations de toutes natures et les réseaux sociaux et interorganisationnels qui ont une incidence locale. Elles s'adressent autant aux valeurs qu'aux comportements.

- Multipartenaires : avec les quatre intentions précédentes, tous les acteurs locaux sont potentiellement interpellés. La revitalisation souhaite que cette interpellation se traduise par un engagement; tous devraient être partie prenante et apporter une contribution.

Cette maxime des $5 \mathrm{M}$ rend compte d'une première dimension de l'intégration dans ces démarches, qui 
qualifie leur caractère global ou holistique. L'intégration signifie l'inclusion des préoccupations sur tous les éléments de la vie locale. Mais une seconde dimension est aussi présente. L'intégration répond à une ambition de cohérence entre ces éléments; elle part de postulats plus ou moins explicites sur l'interdépendance dans les évolutions sectorielles et sur la possibilité de susciter une complémentarité dans les contributions.

L'intégration de la démarche dans sa double dimension est considérée comme nécessaire pour atteindre les résultats attendus d'intégration urbaine et sociale. Elle relève donc d'une cohérence instrumentale. Mais elle est aussi valorisée en tant que telle; elle se considère alors comme une expérimentation de nouveaux modes de gouvernance urbaine ou même comme prototype d'une nouvelle gouvernance. Plusieurs analyses la questionnent sous cet angle.

Dans cet article, nous n'interrogeons pas les postulats et la performance au plan des résultats attendus, ni l'influence réelle du degré d'intégration dans la démarche sur les résultats. Nous limitons l'exposé à l'intégration dans la démarche.

\section{Les modalités de l'intégration dans la démarche de revitalisation}

L'intégration se déploie concrètement par la constitution de partenariats. Comme les études de certaines expériences et les analyses comparatives le montrent, ces partenariats sont extrêmement diversifiés. À l'intérieur d'une même municipalité et d'un même programme (c'est le cas à Montréal), ils peuvent prendre des formes bien différentes, reflétant les multiples configurations locales des forces externes et des rapports entre les acteurs locaux. Ces partenariats peuvent présenter des différences sur plusieurs plans.

- Leur composition : plus ou moins larges, ils rassemblent en proportion très variable des représentants des sphères publique, associative et privée; les secteurs de nature sociale sont souvent plus présents que les secteurs économiques; et les entreprises privées paraissent les plus difficiles à mobiliser. La position de chaque représentant au sein de son organisation est aussi très variable.

- Leur statut : certains partenariats de revitalisation sont créés par obligation; leur création fait partie des exigences des programmes ou des politiques (par exemple les Local Strategic Patnerships en Angleterre). D'autres constituent plutôt des rassemblements informels de diverses organisations du quartier; plusieurs initiatives de revitalisation d'origine communautaire sont de ce type. Le partenariat se formalise davantage lorsqu'il donne naissance à un nouvel organisme autonome dont l'instance dirigeante est composée des différents partenaires; mais il reste aussi parfois au stade du réseau informel entre intervenants locaux.

- Leur mission: sur ce plan aussi l'éventail des expériences est très large; le partenariat peut être centré sur un ou des projets ou sur les services offerts ou sur tout le développement du quartier. Il se limite à l'élaboration de prises de position ou s'étend à la mise en œuvre des orientations et des projets.

- Leur structure et les règles de fonctionnement : certains partenariats ne fonctionnent qu'en réunion générale de tous les partenaires, alors que d'autres se dotent d'instances plus restreintes de coordination et/ou de décision. Les règles de fonctionnement ne sont pas toujours explicitées, surtout lorsque le consensus est la pratique la plus valorisée. L'expression des rapports de force entre les groupes du milieu local est alors plus difficilement décodable.

- La nature des engagements entre les partenaires: dans le cadre d'opérations de revitalisation, les relations verticales entre les différents échelons de gouvernement prennent souvent la forme d'ententes contractuelles. Le financement des gouvernements supérieurs est lié à la réalisation de certains objectifs, parfois formulés de manière large, parfois opérationnalisés par des indicateurs chiffrés. Par contre, les engagements dans les relations horizontales entre partenaires locaux paraissent souvent moins formalisés et relèvent plus du gentleman agreement et des échanges de bons procédés.

- Leurs produits : ils sont de plusieurs ordres. Une première catégorie de produits est facile à repérer : les partenariats génèrent des visions ou des plans de développement plus ou moins englobants, conçoivent et/ou réalisent des projets; ces réalisations peuvent être appréciées de l'extérieur. D'autres réalisations sont moins visibles; elles se manifes- 
tent à l'intérieur du partenariat ou à travers les réalisations des partenaires. Une confiance mutuelle peut se développer au fur et à mesure des réussites. Certaines réalisations sectorielles s'alignent davantage sur les objectifs communs parce qu'une coordination s'est effectuée à travers les échanges au sein du partenariat. Enfin, un autre ordre de produits réside dans les fonctions sociétales que de tels partenariats peuvent remplir; à cet égard, Benington en souligne trois : la légitimation de l'action publique, le partage des risques et des coûts de l'innovation et la capacité de résoudre des problèmes complexes ${ }^{8}$.

Cette très grande diversité des formes de partenariats locaux mis en œuvre dans les opérations de revitalisation urbaine rend difficile l'évaluation de l'intégration qui s'effectue réellement dans l'action publique locale. Cette intégration est susceptible de se manifester à bien des degrés. Cinq-Mars et Fortin distinguent par exemple trois niveaux d'action concertée : l'échange d'informations, de clientèles; la coordination des activités; la coordination des partenaires autour d'une mission, d'un projet commun ${ }^{9}$. Dans ses résultats, l'intégration peut se mesurer ultimement par les réalisations communes, par l'engagement de chaque partenaire à viser les objectifs communs en modifiant concrètement ses actions courantes et par l'absence au niveau local d'effets contradictoires des diverses actions gouvernementales.

Au plan du discours, de la présentation officielle de la revitalisation urbaine, l'intégration prend une place importante et se veut une marque distinctive par rapport aux générations antérieures d'interventions sur ces quartiers. Dans les expériences concrètes, elle inspire la mise en place de partenariats de multiples formes qui tentent de regrouper les citoyens et les acteurs locaux des quartiers défavorisés ainsi que les acteurs externes qui ont un intérêt dans ces quartiers. Il s'agit donc surtout d'une forme d'intégration par la constitution de réseaux. L'État s'estime incapable de faire converger d'autorité l'ensemble des actions des intervenants.

Quant à l'intégration par le marché, elle souffre précisément de sérieuses limites dans ces quartiers compte tenu de leurs caractéristiques socio-économiques, même si la revitalisation vise notamment, et parfois au premier chef, à réinsérer ces quartiers dans les circuits de marché sur tous les plans.
L'intégration par les réseaux se trouve fortement dépendante du fonctionnement de ces réseaux. À cet égard, de nombreuses analyses ont mis en évidence les difficultés de fonctionnement des partenariats et les obstacles à l'intégration.

\section{Cette très grande diversité des formes de partenariats locaux mis en œuvre dans les opérations de revitalisation urbaine rend difficile l'évaluation de l'intégration qui s'effectue réellement dans l'action publique locale.}

\section{Les obstacles à l’intégration locale}

Ces obstacles sont de plusieurs ordres. Certains ont trait à la nature et au fonctionnement des réseaux de partenariat; d'autres, tout en influençant le fonctionnement des partenariats locaux, relèvent de problèmes plus conventionnels de dynamique interorganisationnelle ou du jeu politique local. Tous ces obstacles sont souvent par ailleurs renforcés et davantage enchevêtrés par le découpage des zones d'intervention.

Les partenariats locaux pour fins de revitalisation, dont la forme est très variable comme nous l'avons vu dans la section précédente, sont souvent animés d'une mouvance continuelle et se maintiennent dans un état d'ambiguïté sur le statut de chaque membre et même sur les objectifs. Roulement parmi les représentants des partenaires, différences dans leur statut et dans leur capacité d'engager leurs organisations, caractère central ou périphérique des enjeux du quartier par rapport à la mission de leur organisation sont quelquesuns des facteurs additionnels qui expliquent la difficulté pour les réseaux locaux de mobiliser les partenaires sur de longues périodes.

Par ailleurs, comme l'approche intégrée caractéristique de la nouvelle revitalisation est aussi valorisée dans d'autres démarches sectorielles, il en résulte parfois, au niveau local, un foisonnement de partenariats dont les membres (individus ou organisations) se recoupent largement.

Cet enchevêtrement apparaît davantage comme de la fragmentation plutôt que de l'intégration; cependant, les personnes qui se retrouvent simultanément dans plusieurs de ces partenariats ont sans doute l'oppor- 
tunité de se faire une tête sur l'ensemble des enjeux et des actions locales (forme d'intégration intuitive).

Ces partenariats regroupent surtout des organisations de toutes natures (quelques citoyens peuvent y participer à titre individuel). Les difficultés habituelles dans les relations interorganisationnelles s'y manifestent donc. Les cultures organisationnelles et professionnelles, souvent avec leurs jargons spécialisés, rendent difficile la compréhension commune des problèmes et des objectifs. Les cycles budgétaires et les contraintes d'utilisation des ressources sont variables, entraînent des difficultés d'harmonisation et obligent certains partenaires (surtout associatifs) à faire preuve de grande flexibilité et de capacité d'ajustement pour garder une certaine continuité d'action.

\section{L'exercice du rôle d'élu local dans ces expériences de revitalisation multisectorielle s'avère délicat.}

En outre, les membres des organisations participant à quel que titre que ce soit au partenariat peuvent se retrouver dans des dilemmes de priorité d'allégeance qui se traduisent concrètement dans les choix d'activités et d'allocation des ressources humaines, matérielles et financières. La question centrale qui se pose aux organisations peut alors se formuler ainsi : est-ce que la revitalisation urbaine intégrée demande des efforts en termes d'importance relative et en marge des activités courantes quant à la nature des contributions? Ou suppose-t-elle de réorienter et de faire autrement les activités normales ? Ou nécessite-t-elle une combinaison de ces deux stratégies? La réponse à cette question conditionne non seulement l'efficacité à court terme de la contribution organisationnelle, mais aussi la capacité de maintenir à long terme des améliorations, une fois que cessent les mesures particulières du programme de revitalisation. Cette question renvoie aux débats dans les programmes de revitalisation sur le mainstreaming en Angleterre ou sur le retour au droit commun en France. Le processus d'allocation des ressources est central dans la revitalisation puisque l'état des quartiers exige des réinvestissements massifs. Et le degré de réallocation des ressources propres de chaque organisation partenaire en fonction des projets communs de revitalisation pourrait être l'un des indicateurs du degré d'intégration réelle de l'action publique locale. L'existence de fonds spéciaux pour la revitalisation, en sus des ressources de chaque organisation, ne garantit pas une intégration des actions de tous; elle peut la faciliter si l'utilisation de ces fonds par les partenaires est conditionnelle à des changements de façons de faire et pas seulement à l'ajout d'activités; sinon, elle risque de servir à compenser des coupures ou à faciliter le financement d'autres activités. Par ailleurs, l'allocation de ces fonds spéciaux s'inscrit d'emblée dans le jeu politique local.

Ce jeu se déroule sur plusieurs plans : dans la sphère politique proprement dite, entre les sphères (notamment entre le politique et l'associatif); il s'enracine par ailleurs dans l'évolution des rapports de force entre groupes locaux entraînée par la transformation des quartiers. Les opérations de revitalisation d'initiative gouvernementale impliquent les élus des divers niveaux de gouvernement qui participent au financement. Dans les expériences de revitalisation d'origine communautaire comme les Comprehensive Community Initiatives, l'importance des élus est moins grande sans que la dimension politique ne soit absente pour autant. L'intérêt des élus peut différer selon la place des quartiers ciblés dans leur électorat et des questions d'exclusion dans l'opinion publique. Les élus locaux sont habituellement davantage interpellés sur deux plans. D'abord, par rapport au quartier ciblé, l'allocation des ressources additionnelles peut être une occasion de consolider leurs appuis locaux, et leurs relations personnelles peuvent influencer le fonctionnement du réseau de partenariat. D'autre part, par rapport aux paliers supérieurs de gouvernement, ils doivent, en plus de jouer leur rôle traditionnel d'intercesseur, apprendre un autre rôle, celui de combiner divers programmes gouvernementaux pour financer les projets de revitalisation; le succès de plusieurs opérations semble en partie dû à l'habileté combinatoire des élus et des responsables locaux et à leur facilité de conjuguer divers types d'aide.

L'exercice du rôle d'élu local dans ces expériences de revitalisation multisectorielle s'avère cependant délicat. Plusieurs analyses d'expériences, surtout lorsque le partenariat est davantage formalisé, soulignent que les élus éprouvent souvent un inconfort, un malaise à œuvrer dans un tel contexte; on s'attend à ce qu'ils fassent preuve de leadership, mais en même temps à ce qu'ils n'interviennent pas indûment dans la dynamique du partenariat. Souvent, la tension la plus res- 
sentie se situe entre l'élu et les associations et groupes communautaires; elle dénote une compétition pour la légitimité. L'élu s'appuie sur la légitimité démocratique, mais souvent à partir d'un territoire plus vaste, alors que les groupes communautaires, bien implantés dans la zone d'intervention, revendiquent une meilleure connaissance des réalités locales et des besoins. Le pilotage de l'opération de revitalisation suppose à la fois affirmation de pouvoir et négociations plus ou moins explicites.

\section{Dans bon nombre d'expériences, la revitalisation qui s'annonce comme globale devient dans les faits marginale et spécialisée.}

Ces négociations peuvent être incontournables et ardues ou ne pas l'être selon la nature des clivages au sein de la population locale. Or, la revitalisation induit souvent de tels clivages, surtout lorsqu'un de ses objectifs est de transformer la composition sociale du quartier par l'arrivée de catégories sociales moins défavorisées. Les nouveaux venus n'ont pas forcément les mêmes besoins ni les mêmes comportements civiques et peuvent exprimer une vision du quartier bien différente en privilégiant une accélération du redéveloppement. L'élu local doit alors assumer (pour les matières qui relèvent de sa compétence) un rôle de facilitateur ou d'arbitre entre les anciens et les nouveaux, ce qui met à l'épreuve sa propension habituelle à jouer la carte du consensus. Dans ces cas, l'intégration du quartier au reste de la ville que recherche la revitalisation passe par une forme de désintégration du quartier, et l'élu local (et son administration) contribue au maintien d'une certaine cohésion locale; mais son influence est limitée par le champ de compétence municipale.

Tous ces obstacles à l'intégration de l'action publique dans les opérations de revitalisation, qu'ils proviennent de la gouvernance du réseau de partenariat ou du jeu politique local, sont souvent renforcés par les effets du découpage des zones d'intervention. Soucieuse d'efficacité, la revitalisation concentre des ressources spéciales (hors budget courant) sur des zones restreintes, parfois de petite taille (de l'ordre de l'unité de voisinage). Certaines de ces zones ne correspondent ni à des territoires administratifs (circonscription électorale, aire d'organisation de services publics), ni à une réalité sociale locale (identité de quartier, zone d'influence d'associations ou de réseau d'associations). Les partenaires potentiels n'ont alors spontanément qu'un intérêt partiel pour de telles zones, et l'action publique de revitalisation impose de nouvelles priorités, induit de nouveaux mécanismes de coordination qui peuvent alors être perçus comme dérangeants, voire menaçants.

Devant tous ces obstacles mis en évidence par les analyses d'expériences, l'aspiration de la revitalisation à des approches intégrées s'avère très ambitieuse. Elle amène certes parfois des innovations intéressantes et des améliorations concrètes significatives. Mais le grand dessein des $5 \mathrm{M}$ apparaît souvent hors de portée. En conclusion d'une analyse de plusieurs cas, Huxham et Vangen notent que les partenariats ont plus de succès quand ils sont centrés sur une seule question, un seul projet et n'incluent qu'un petit nombre d'organisations ${ }^{10}$ ! Une telle conclusion ne doit cependant pas forcément être généralisée. Tout dépend du plan sur lequel on veut évaluer le succès : amélioration des services, de la qualité du milieu, transformation de la composition sociale, réduction des tensions, cohésion dans l'action publique... Il n'en reste pas moins que la liste des obstacles recensés dans le fonctionnement des expériences est longue et lourde.

Peut-on mettre efficacement en œuvre le grand dessein des $5 \mathrm{M}$ ou celui-ci est-il voué à rester dans le monde de l'utopie, certes inspiratrice ? Dans les faits, plusieurs expériences trouvent des moyens d'atténuer sur un plan opérationnel certains des obstacles mentionnés, surtout lorsque des leaders locaux sont insérés dans des réseaux de relations interpersonnelles assez stables. Mais les innovations concrètes se heurtent à des données structurelles plus incontournables. L'approche intégrée, telle que nous l'avons résumée dans les $5 \mathrm{M}$, pourrait être, à première vue, un vecteur de transformation assez radicale des façons de faire et des institutions qui se diffuserait au-delà des zones d'intervention. Cependant, dans bon nombre d'expériences, la revitalisation qui s'annonce comme globale devient dans les faits marginale et spécialisée en ce qu'elle apporte un supplément temporaire d'interventions ponctuelles sans que les activités normales des différents agents ne soient affectées. Ne faut-il pas alors chercher, au-delà des difficultés opérationnelles $\mathrm{du}$ processus d'intégration, des facteurs plus fondamentaux de blocage des approches intégrées? Une 
transformation de ces facteurs est-elle une condition préalable au plein déploiement des approches intégrées ?

\section{Le « mur » de l’intégration}

Une analogie peut stimuler le démarrage de la réflexion sur ces questions. En aéronautique, les phénomènes liés au dépassement du mur du son obligent à revoir la conception d'ensemble de l'appareil. L'avion qui doit voler à vitesse supersonique ne peut pas avoir les mêmes caractéristiques que celui conçu pour la vitesse subsonique. Il faut en revoir certaines propriétés de base. Dans la revitalisation urbaine, l'intégration ne serait-elle pas une sorte d'équivalent du mur du son ? L'action publique en mode intégrée ne devrait-elle pas avoir une configuration différente ? Si tel devait être le cas, il ne suffirait pas d'articuler autrement les pièces du puzzle local, de seulement aménager les transactions entre sphères et secteurs d'activités ou de coordonner les acteurs organisationnels; il faudrait revoir les découpages entre sphères et secteurs d'activités ainsi que le partage des rôles. Évoquons certains de ces facteurs plus fondamentaux de blocage de l'action intégrée au niveau local: le fractionnement de l'État, les limites de la démocratie représentative et l'obsolescence des champs sectoriels.

La revitalisation urbaine intégrée d'initiative gouvernementale manifeste un paradoxe de fond. L'État qui préconise cette intégration est sans doute le partenaire le moins intégré. Sa fragmentation est telle, verticalement, horizontalement et institutionnellement, que le partenariat visé touche parfois d'abord et surtout les acteurs publics et parapublics. Cette fragmentation est entre autres à l'origine des difficultés pour insérer les activités courantes des services publics dans l'entreprise de revitalisation. De manière bien concrète au plan budgétaire, il est difficile de connaître le niveau des ressources publiques dépensées dans la zone, faute d'une comptabilité publique territoriale englobant tous les niveaux de gouvernement et tous les secteurs d'activité. Comment peut-on, dans de telles conditions, appuyer solidement des priorités locales? Cette absence d'intégration, même minimale, sous forme d'une vue d'ensemble de la présence étatique locale entraîne une dissymétrie entre ce que l'État attend du citoyen et ce que l'État offre au citoyen. L'État s'attend, notamment à l'égard des pauvres des zones de revitalisation, à ce que le citoyen s'intègre personnellement, développe l'ensemble de sa personnalité (employabilité, réseau social, participation civique) pour pouvoir mieux s'insérer dans la vie économique et sociale.

\section{La revitalisation urbaine intégrée d'initiative gouvernementale manifeste un paradoxe de fond.}

De manière plus spécifique, la fragmentation verticale et le partage des responsabilités entre niveaux de gouvernement et entre instances décentralisées et instances déconcentrées ne sont pas sans incidence sur les possibilités d'intégration des actions de revitalisation. Le sort des résidents des zones d'intervention dépend largement de décisions prises au-dessus de ces zones et à différents paliers, dans toutes les sphères, qu'il s'agisse de l'accès aux emplois, aux opportunités urbaines de toutes sortes ou de la localisation de logements sociaux ou de l'obtention de subventions philanthropiques. La fragmentation de l'État rend difficile l'établissement de liens entre les relais à différents niveaux de prise de participation dans les enjeux de quartier. Le recours à diverses modalités de gouvernance via la constitution de partenariats locaux peut être interprété comme la recherche d'un antidote à cette fragmentation. Faute de pouvoir se coordonner, les unités relevant de l'État se feraient coordonner par des tiers de la société civile présents dans les instances partenariales. Ces tiers agiraient comme des agents externes de changement de la machine étatique. Dans les faits, comme l'expérience des Local Strategic Partnerships anglais le montre du moins jusqu'à maintenant, cette capacité de changement ne doit pas être surestimée.

L'expérience des partenariats locaux de revitalisation soulève une autre question de fond, effleurée dans la section précédente par son symptôme au plan opérationnel : le malaise des élus locaux à œuvrer dans ce genre d'entreprise collective. Cette question touche à la conciliation, voire à la compatibilité entre la démocratie représentative et la démocratie participative. Les présentations officielles de la revitalisation insistent sur la participation des citoyens, conformément aux tendances des politiques sociales actuelles qui préconisent l'activation des citoyens (la revitalisation est d'ailleurs parfois incluse dans les politiques socia- 
les). Cette participation est recherchée tant au plan individuel (insertion dans des activités de formation, contribution bénévole à des projets) qu'au plan collectif (prise en charge des problèmes locaux par la population locale). La revitalisation vise alors l'empowerment local, cherche à développer la capacité du milieu local (organisations, associations et citoyens) à identifier les problèmes, à définir des solutions et à les mettre en œuvre. Dans des termes dont l'emploi se généralise, la revitalisation se donne pour objectif de développer le capital social collectif du milieu.

$\mathrm{Si}$, conformément aux vœux officiels, tous les citoyens étaient entièrement activés sur tous les plans, ils mettraient en œuvre une nouvelle forme de démocratie directe participative qui aurait deux caractéristiques importantes. D'une part, les décisions concernant le sort du quartier et leur sort individuel seraient entre leurs mains; d'autre part, ils assumeraient une bonne partie des services collectifs locaux puisqu'on leur demande de prendre des initiatives, ce qu'ils font d'ailleurs souvent. Dans un tel scénario, quelle place reste-t-il à la démocratie représentative, aux élus et aux machines administratives prestataires de services qu'ils commandent? Et quels nouveaux mécanismes d'imputabilité sont à mettre en place ? Cependant, sur le plan de la démocratie comme sur celui de la coordination étatique, la lettre des discours officiels de la revitalisation évoque plus de transformations que ce qu'elle inspire réellement sur le terrain. Et les effets ne sont pas toujours ceux attendus. L'empowerment local qui, au niveau des objectifs, laisse entrevoir une forme d'autonomie plus grande se révèle parfois dans les faits instrumentalisé au profit des institutions existantes. Le potentiel de transformation que certaines présentations mettent dans la revitalisation ne semble s'actualiser que timidement.

Ce diagnostic s'applique aussi à l'ambition multisectorielle. Certes, habituellement, dans les expériences de revitalisation, de nombreux secteurs se mettent en action. Parfois, chacun y va de son propre dispositif « intégré » et met en place sa propre structure de partenariat. La simultanéité des actions sectorielles ne garantit cependant pas leur convergence vers la réalisation d'objectifs communs, même si elle peut y contribuer, surtout lorsqu'elle a été concertée. Un cran additionnel dans l'intégration est franchi lorsque des initiatives se développent dans les interstices entre secteurs, de façon autonome ou avec la collaboration des secteurs proches (par exemple des projets d'alimentation des élèves entre santé et éducation). En jouant sur les interstices, de telles initiatives ne remettent pas en cause les découpages sectoriels ni les champs de pratiques professionnelles spécialisées qui s'y développent institutionnellement.

Une telle remise en cause s'affirmerait sans doute si la logique d'intégration était poussée plus loin en fonction des grands objectifs de revitalisation (activation des citoyens, sécurité, animation du milieu...). Prenons l'exemple de la formation. Bon nombre d'opérations de revitalisation visent à réinsérer les citoyens des zones ciblées sur le marché du travail; les mesures pour améliorer l'employabilité y sont vigoureuses, mais elles peuvent être menées indépendamment des initiatives du secteur de l'éducation ou de celles du milieu associatif qui cherchent à développer des habiletés sociales utiles en milieu de travail. Dans la mutation de l'État-providence, qui cherche plutôt à investir dans le développement des citoyens qu'à les assister, la formation est susceptible de requestionner le partage des responsabilités et les limites des secteurs. La logique de l'ambition des $5 \mathrm{M}$ de la revitalisation peut amener de tels questionnements même si, dans la pratique, cette ambition se trouve souvent phagocytée, dévitalisée par le jeu des institutions et des organisations en place.

La revitalisation urbaine intégrée ne pourra sans doute pleinement produire ses effets positifs attendus que si elle dépasse le mur de l'intégration. À cette fin, elle doit contribuer à des changements structurels plus fondamentaux dans la composition de l'État, dans le renouvellement de la démocratie et dans la division institutionnelle du travail sectoriel. De tels changements s'expérimentent avant de s'institutionnaliser. À cet égard, les expériences de revitalisation sont des laboratoires intéressants de nouvelles formules, même si leur généralisation à plus grande échelle peut connaître tous les aléas de l'innovation. L'intégration locale n'est pas mécanique; elle ne se résume pas à une combinaison additive de forces externes ni à un assemblage de mesures conçues centralement. Pour réussir - et c'est la piste de réflexion que nous inspirent les bilans effectués jusqu'à maintenant -, elle doit remettre en cause certains de ces éléments externes.

\section{Notes et références}

1 Gérard Divay est professeur à l'INRS Urbanisation, Culture et Société. Ses travaux de recherche portent actuelle- 
ment entre autres sur les approches intégrées de gestion locale et sur les politiques territorialisées.

2 Boucher, J.L. et L. Favreau (1998). « Le développement social urbain. L'avenir de la revitalisation économique et sociale des quartiers en difficulté », Économies et Solidarités, vol. $29, \mathrm{n}^{\circ} 2$.

3 Certaines expériences sont présentées dans le numéro de novembre 2004 de la revue Urbanité.

4 Quartiers en crise. Approches intégrées de développement urbain.

http:// www.globenet.org/horizon-local/shelter/

integurb.html (consultation: 13 janvier 2005).

5 De Decker, P., J. Vranken, J. Beaumont et I. Van Nieuwnhuyze (dir.) (2003). On the Origins of Urban Development Programmes in Nine European Countries, Antwerp, Garant, p. 183.

6 Geddes, M. et J. Benington (2001) (dir.). Local Partnerships and Social Exclusion in the European Union, London, Routledge, chap. 1.
7 Ces interactions sont questionnées dans le rapport de recherche sur les expériences de la Ville de Montréal fait pour le compte de la Ville de Montréal. Divay, G., P. Bernard, P.J. Hamel, D. Rose, G. Sénécal et A.-M. Séguin avec la collaboration de B. Charbonneau, G. Côté et P. Herjean (2004). Projet pilote de revitalisation intégrée. Démarche d'évaluation, Montréal, INRS urbanisation, Culture et Société, 249 p.

8 Benington, J. (2001). « Partnerships as Networked Governance. Legitimation, Innovation, Problem-solving and Coordination », dans M. Geddes et J. Benington, op.cit., chap. 10

9 Cinq-Mars, M. et D. Fortin (1999). «Perspectives épistémologiques et cadre conceptuel pour l'évaluation de l'implantation d'une action concertée », The Canadian Journal of Program Evaluation, vol. 13, n 2, p. 29-54.

10 Huxham, C. and S. Vangen (2000). « What Makes Partnerships Work? », in S.P. Osborne,. (dir.). Public Private Partnerships, London et New York, Routledge, p. 293310 . 


\section{Publicité}

« Revue Organisations »

Site Web 